\section{Réplica: Opinión del Comité Consultivo de Inmunizaciones, Sociedad Chilena de Infectología}

\section{Reply: Position of the Advisory Committee on Immunizations, Chilean Infectious Diseases Society}

\section{Sr. Editor:}

En respuesta a la carta de Lepetic y colaboradores, en la cual discuten y critican nuestra preferencia como Comité Consultivo de Inmunizaciones de la SOCHINF por la vacuna antineumocócica conjugada 13 valente (PCV13) en lugar de la vacuna 10 valente (PCV10) ${ }^{1}$, quisiéramos precisar los siguientes puntos:

Concordamos en que la introducción de PCV10 en Chile, y su posterior incorporación como vacuna programática en 2011, ha sido una medida de salud pública muy beneficiosa e incuestionable a la luz de los datos de vigilancia disponibles que muestran un claro descenso en las enfermedades neumocócicas invasoras (ENI), neumonías y muertes por neumonias en menores de 2 años ${ }^{2,3}$. Sin embargo, el impacto de las vacunas neumocócicas conjugadas que contienen múltiples serotipos debe ser evaluado también considerando la mejor cobertura de serotipos circulantes y emergentes en cada región. En Chile, los serotipos que más han aumentado son el 3 y 19A no contenidos en PCV10, y concordamos con los autores en que el serotipo 3 ha sido difícil de reducir con las vacunas disponibles; sin embargo, la situación del serotipo 19A es completamente distinta. Si bien existe información inmunológica de protección cruzada de PCV10 contra el serotipo 19A, y autoridades regulatorias de varios países han registrado esta indicación, la evidencia clínica y epidemiológica en nuestro país indica que esta protección cruzada no está ocurriendo habiendo transcurrido 5 años desde la incorporación de PCV10 en nuestro Plan Nacional de Inmunizaciones. Al respecto, existe información reciente que complementa los datos epidemiológicos chilenos que mencionamos en nuestra publicación y que confirman que entre enero 2014 y junio de 2016 se han registrado 105 menores de 6 años con ENI por serotipo $19 \mathrm{~A}^{4}$. De ellos hay 94 niños con datos de vacunación disponible, de los cuales solo 2/94 no tenían ninguna dosis de vacuna, 28/94 (30\%) tenían 2 dosis y el 63/94 (67\%) tenían esquema completo de 2 dosis +1 refuerzo. En esta serie se registraron incluso dos niños fallecidos ${ }^{4}$.

En relación a la protección indirecta de grupos no vacunados otorgada por las vacunas neumocócicas con- jugadas, que consideramos en Chile ha sido insuficiente, si bien es cierto que en algunos países que usan PCV13 no se ha mostrado impacto, como por ejemplo Francia o Noruega ${ }^{5}$ en la gran mayoría de ellos el efecto ha sido notable ${ }^{5-8}$. En cambio en países que utilizan PCV10 el efecto ha sido más discreto y muy variable de una región a otra. Así en Finlandia, país que usa PCV10 en esquema $2+1$ en niños desde 2011, se evidencia efecto sobre ST contenidos en PCV10 en población no vacunada pero a partir del año 2014 se observa un aumento de casos por serotipos no contenidos pero relacionados lo que finalmente mantiene en mayores de 64 años números absolutos de ENI sin modificaciones, con 279 casos como promedio anual entre los años 2004-2010 y 348 casos promedio entre 2011-2015 . Por otra parte en Brasil, país que también usa PCV10 muestra impacto, en algunas regiones, en ENI en individuos sobre 65 años $^{10}$ pero otras no ${ }^{10-12}$. En gran medida el efecto se ve reducido por el aumento de serotipos 19A, 6 A y $6 \mathrm{C}$ lo que es explicable por el escaso efecto de PCV10 en portación nasofaríngea de estos ST no incluidos en la formulación de PCV10 ${ }^{14}$. Esto puede también explicar lo que ocurre en Chile por lo que parece muy razonable el optar por una vacuna que sí ha mostrado en forma consistente una reducción de carga de enfermedad neumocócica en adultos y adultos mayores ${ }^{5-7}$.

Tal como precisan Lepetic y cols el efecto global de estas vacunas es indiscutible, pero también concordamos con lo que señala la OMS de que si bien PCV10 y PCV13 tienen perfiles de seguridad y eficacia similar para los serotipos contenidos en cada vacuna, la elección de la vacuna neumocócica conjugada dependerá de factores como los serotipos contenidos en las vacunas y los prevalentes en los grupos objetivo ${ }^{15}$. De modo que existiendo en nuestro país casos de niños vacunados adecuadamente con PCV10 que han presentado infecciones invasoras graves por el serotipo 19A, y disponiendo de una vacuna que brinda protección contra dicho serotipo, consideramos técnica y éticamente mandatorio proponer un cambio desde PCV10 a PCV13. Los programas de inmunización modernos han de ser dinámicos, vale decir, deben ser revisados periódicamente a la luz de la nueva evidencia disponible y de la información epidemiológica local. Este proceder permite proponer cambios en un sentido que consideramos correcto.

\section{Marcela Potin y Jaime Cerda en representación del Comité Consultivo de Inmunizaciones, SOCHINF.}

Fuentes de financiamiento: Ninguna Conflictos de interés: Ninguno para la vacuna en cuestión. 


\section{Referencias bibliográficas}

1.- Potin M, Fica A, Wilhem J, Cerda J, Contreras L, Escobar C, et al. Opinión del Comité Consultivo de Inmunizaciones Sociedad Chilena de Infectología. Vacuna neumocóccica conjugada en niños y la emergencia de serotipo 19A. Rev Chil Infectol. 2016; 33: 304-6.

2.- Díaz J, Terrazas S, Bierrenbach A L, Toscano C M, Alencar G P, Álvarez A, et al. Effectiveness of the 10-valent pneumococcal conjugate vaccine $(\mathrm{PCV}-10)$ in children in Chile: a nested case-control study using nationwide pneumonia morbidity and mortality surveillance data Disponible en: PLoS ONE 2016; 11: e0153141.

3.- Fernandez V J, Goecke H C, von Borries C, Tapia RN, Santolaya de P M. Incidencia de egresos por neumonía en niños menores de 24 meses antes y después de la implementación de la vacuna conjugada antineumocócica 10-valente en el Programa Nacional de Inmunizaciones de Chile. Rev Chil Pediatr. 2015; 86: 168-72.

4.- González C, Burgos P, Brstilo I, Hormazábal J C, Araya P, Alarcón P, et al. Estatus vacunal de niños de hasta 5 años de edad con aislamiento de serotipo 19A entre 2014-2016 Comunicación oral XXXIII Congreso Chileno de Infectología Valdivia,Chile, 12 a 15 de octubre 2016.

5.- $\quad$ Tin Tin Htar M, Christopoulou D, Schmitt H-J. Pneumococcal serotype evolution in Western Europe. BMJ Infectious Diseases $2015 ; 15: 419$

6.- Pilivishli T, Gierke R, Farley M, et al. Direct and Indirect Impact of 13-Valent Pneumococcal Conjugate Vaccine (PCV13) use on Invasive Pneumococcal Disease (IPD) among Children and Adults Session: Oral Abstract Session: Vaccines, Vaccine Preventable Disease, and their Impact ID Week, New Orleans USA october 2016

7.- Moore M, Link-Gelles R, Shaffner W, Lynfield R, Holtzman C, Harrison L H, et al Effectiveness of 13-valent pneumococcal conjugate vaccine for prevention of invasive pneumococcal disease in children in the USA: a matched case-control study. Lancet Respir Med 2016; 4: $399-406$
8.- Chalmers J D, Campling J, Dicker A, Woodhead M, Madhava H. A systematic review of the burden of vaccine preventable pneumococcal disease in UK adults. BMC Pulmonary Medicine 2016; 16: 77-87.

9.- National Institute for Health and Welfare. Incidence of pneumococcal disease in Finland. Disponible en: www. thl.fi/en/web/thlfi-en/research-and-expertwork/projectsand-programmes/monitoring-the-population-effectivenessof-pneumococcal-conjugate-vaccination-in-the-finnishnational-vaccination-programme/incidence-of-invasivepneumococcal-disease-in-finland (acceso el 28 noviembre de 2016).

10.- Moreira M, Cintra O, Harriague J, Hausdorf W P, Hoet B. Impact of the introduction of the pneumococcal conjugate vaccine in the Brazilian routine childhood national immunization program. Vaccine 2016; 34: 2766 -78

11.- Dos Santos S R, Passadore L F, Takagi E H, Fujii C M, Yoshioka C R, Gilio A E, et al. Serotype distribution of Streptococcus pneumoniae isolated from patients with invasive pneumococcal disease in Brazil before and after ten-pneumococcal conjugate vaccine implementation. Vaccine 2013; 31: 6150-4

12.- Caierao J, Hawkins P, Sant'anna F H, da Cunha G R, d'Azevedo P A, McGee L, et al. Serotypes and genotypes of invasive Streptococcus pneumoniae before and after PCV10 implementation in southern Brazil. PLOS ONE 2014; 9: e111129.

13.- Andrade A L, Minamisava R, Policena G, Cristo E B, Domingues C M, de Cunto Brandileone M C, et al. Evaluating the impact of PCV-10 on invasive pneumococcal disease in Brazil: a time-series analysis. Hum Vaccin Immunother 2016;12: 285-92.

14.- Brandileone M C de C, Zanella R, Almeida S C G, Brandao A P, Ribeiro A F, Carvalhanas T R, et al. Effect of 10-valent pneumococcal conjugate vaccine on nasopharyngeal carriage of Streptococcus pneumoniae and Haemophilus influenzae among children in São Paulo, Brazil. Vaccine 2016; 34: 5604-11.

15.- Pneumococcal vaccines WHO position paper-2012 Disponible en: http://www.who.int/wer/2012/wer8714.pdf (acceso el 25 noviembre de 2016). 